\title{
Existence and convergence results of meromorphic solutions to the equilibrium system with angular velocity
}

Bo Meng ${ }^{1 *}$

"Correspondence:

bo.mengsd@gmail.com ${ }^{1}$ College of Mathematics and

Systems Science, Shandong

University of Science and

Technology, Qingdao, China

\section{每 Springer}

\begin{abstract}
We study the equilibrium system with angular velocity for the prey. This system is a generalization of the two-species equilibrium model with Neumann type boundary condition. Firstly, we consider the asymptotical stability of equilibrium points to the system of ordinary differential equations type. Then, the existence of meromorphic solutions and the stability of equilibrium points to the system of weakly coupled meromorphic type are discussed. Finally, the existence of nonnegative meromorphic solutions to the system of strongly coupled meromorphic type is investigated, and the asymptotic stability of unique positive equilibrium point of the system is proved by constructing meromorphic functions.
\end{abstract}

Keywords: Asymptotic stability; Equilibrium system; Meromorphic function

\section{Introduction}

The equilibrium system with angular velocity is noted for its pattern-forming behavior and has been widely used as a model for the study of obstacle problems involving reservoir simulation. These include the effects of noise on bifurcations, pattern selection, spatiotemporal chaos, and the dynamics of defects; see, for example, [1-8] and the references therein for details. It also has been used to model patterns in simple fluids and in a variety of complex fluids and biological materials, such as neural tissue [3, 7]. These problems are widely studied and very well used in many areas of mathematics and physics, see $[3,5,6$, 9]. Since it was initiated by Paul Dirac in order to get a form of quantum theory compatible with special relativity, the Dirac equation has been playing a critical role in some fields of mathematics and physics, such as quantum mechanics, Clifford analysis, and partial differential equations.

As one of the universal equilibrium systems used in the description of pattern formation in spatially extended dissipative systems, the general equilibrium differential equation can also be found in the study of convective hydrodynamics, plasma confinement in toroidal devices, viscous film flow, and bifurcating solutions of the modified equilibrium differential equation [6, 10, 11]. In recent years, some references such as Sheng et al. [12], Zhai et al. [13], Zhang [14], Wu et al. [15], Sun et al. [16], Li et al. [17], Bai and Sun [18], Wang et al. [19], and so on, introduced many beautiful patterns to satisfy practical requirements of modern computing systems with

(c) The Author(s) 2019. This article is distributed under the terms of the Creative Commons Attribution 4.0 International License (http://creativecommons.org/licenses/by/4.0/), which permits unrestricted use, distribution, and reproduction in any medium, provided you give appropriate credit to the original author(s) and the source, provide a link to the Creative Commons license, and indicate if changes were made. 
multi-processors. There is the potential of considering the linearization characteristics to be further developed for the system of equilibrium boundary value problems. Ardila [20] studied the existence and stability of standing waves solutions of a threecoupled nonlinear Schrödinger system related to the Raman amplification in a plasma. $\mathrm{Hu}$ and Yin [21] considered dynamics of the compressible Navier-Stokes equation in one spatial dimension for a viscous fluid with vanishing thermal conductivity. For the case of ideal polytropic gases, it is shown that the rarefaction waves in this medium are stable with regards to sufficiently weak perturbations of the velocity and pressure fields.

Motivated and inspired by the references [18-21], in this paper we further consider the following universal equilibrium equations with nondifferentiable boundary conditions:

$$
\begin{aligned}
& -\Delta u+u=a(|x|)|u|^{p-2} u, \quad x \in B_{1} \\
& u>0, \quad x \in B_{1}, \\
& \frac{\partial u}{\partial v}=0, \quad x \in \partial B_{1},
\end{aligned}
$$

where $B_{1}$ is the unit ball centered at the origin in $\mathbb{R}^{n}, n \geq 3$ and $p>2$ and $a \in L^{1}(0,1)$ is increasing, not constant and $a(r)>0$ a.e. in $[0,1]$.

Meanwhile, we are also interested in the equilibrium elliptic system given by

$$
\begin{aligned}
& -\Delta u+u=f_{u}(|x|, u, v), \quad x \in B_{1} \\
& -\Delta v+v=f_{v}(|x|, u, v), \quad x \in B_{1} \\
& \frac{\partial u}{\partial v}=\frac{\partial u}{\partial v}=0, \quad x \in \partial B_{1},
\end{aligned}
$$

under suitable assumptions on $f$. Our assumptions do allow some supercritical nonlinearities. Problems in this abstract form are often referred to as equilibrium problems. More details on this problem class can be found in $[9,22]$.

Let $I=\{1,2, \ldots, n\}$ be an index set, $H_{i}$ be a real Hilbert space with inner product $\langle\cdot, \cdot\rangle_{i}$ and norm $\|\cdot\|_{i}$, respectively. Let $A: H_{1} \rightarrow H_{1}, B: H_{2} \rightarrow H_{2}, F_{1}: H_{1} \times H_{2} \rightarrow H_{1}$, and $\eta_{1}: H_{1} \times H_{1} \rightarrow H_{1}$ be mappings. Let $a_{i}: H_{i} \times H_{i} \rightarrow \mathbb{R}$ be a coercive continuous map such that

(C1) $a_{i}\left(\sigma_{i}, \sigma_{i}\right) \geq c_{i}\left\|\sigma_{i}\right\|_{i}^{2}$

(C2) $\left|a_{i}\left(\varrho_{i}, \sigma_{i}\right)\right| \leq d_{i}\left\|\varrho_{i}\right\|_{i} \cdot\left\|\sigma_{i}\right\|_{i}$

for any $\varrho_{i}, \sigma_{i} \in H_{i}$.

Let $b_{i}: H_{i} \times H_{i} \rightarrow \mathbb{R}$ be a map with nondifferentiable terms such that

(C3) $b_{i}$ is a linear function for the first variable;

(C4) $b_{i}$ is a convex function;

(C5) There exists a positive constant $\gamma_{i}$ satisfying

$$
\gamma_{i}\left\|\varrho_{i}\right\|_{i} \cdot\left\|\sigma_{i}\right\|_{i} \geq a_{i}\left(\varrho_{i}, \sigma_{i}\right)
$$

for any $\varrho_{i}, \sigma_{i} \in H_{i}$. 
(C6)

$$
b_{i}\left(\varrho_{i}, \sigma_{i}-w_{i}\right) \geq b_{i}\left(\varrho_{i}, \sigma_{i}\right)-b_{i}\left(\varrho_{i}, w_{i}\right)
$$

for any $\varrho_{i}, \sigma_{i}, w_{i} \in H_{i}$.

Based on the above notations, we then define the proposed system of generalized nonlinear variational inequality problems as follows:

Find $(x, y) \in H_{1} \times H_{2}$ such that

$$
\left\{\begin{array}{l}
\left\langle F_{1}(A x, B y)-f_{1}, \eta_{1}\left(\sigma_{1}, x\right)\right\rangle_{1}+a_{1}\left(x, \sigma_{1}-x\right)+b_{1}\left(x, \sigma_{1}\right)-b_{1}(x, x) \geq 0 \\
\quad \forall \sigma_{1} \in H_{1}, \\
\left\langle F_{2}(A x, B y)-f_{2}, \eta_{2}\left(\sigma_{2}, y\right)\right\rangle_{2}+a_{2}\left(y, \sigma_{2}-y\right)+b_{2}\left(y, \sigma_{2}\right)-b_{2}(y, y) \geq 0 \\
\quad \forall \sigma_{2} \in H_{2},
\end{array}\right.
$$

where $f_{i} \in H_{i}$ is given for each $i \in I$.

Remark 1 There are some special cases for the model problem (3) (see [23]):

(1) If $A=B=I, f_{i}=0$, and $a_{i}\left(\varrho_{i}, \sigma_{i}\right)=0$, then (3) is equivalent to

$$
\left\langle F_{1}(x, y), \eta_{1}\left(\sigma_{1}, x\right)\right\rangle_{1}+b_{1}\left(x, \sigma_{1}\right)-b_{1}(x, x) \geq 0,
$$

where $\sigma_{1} \in H_{1}$.

$$
\left\langle F_{2}(x, y), \eta_{2}\left(\sigma_{2}, y\right)\right\rangle_{2}+b_{2}\left(y, \sigma_{2}\right)-b_{2}(y, y) \geq 0,
$$

where $\sigma_{2} \in H_{2}$.

(2) If $H_{1}=H_{2}=H, f_{i}=f_{2}=f, \eta_{1}=\eta_{2}=\eta, a_{1}=a_{2}=a, b_{1}=b_{2}=b$, then (3) is reduced to

$$
\langle F(A x, B x)-f, \eta(v, x)\rangle+a(x, v-x)+b(x, v)-b(x, x) \geq 0,
$$

where $v \in H$.

\section{Preliminaries}

Let us recall some basic definitions and lemmas that we need in the forthcoming analysis.

Definition 1 We say that the functional $\Phi$ satisfies the Palais-Smale condition (see [15, 24-26]) if any sequence $\left\{u_{n}\right\}_{n \in \mathbb{N}} \subset X$ has a convergent subsequence provided $\left\{\Phi\left(u_{n}\right)\right\}_{n \in \mathbb{N}}$ is bounded and $\Phi^{\prime}\left(u_{n}\right) \rightarrow 0$ as $n \rightarrow+\infty$.

Definition 2 For any $\lambda>0$, we define the subfunctions associated with the universal equilibrium operator (1) by (see [11])

$$
\mathbb{I}_{+}^{\tau, \lambda} f(x)=\frac{1}{\Gamma(\tau)} \int_{-\infty}^{x} f(\xi)(x-\xi)^{\tau-1} e^{-\lambda(x-\xi)} d \xi
$$

and the subfunctions associated with the universal equilibrium operator (2) by

$$
\mathbb{I}_{-}^{\tau, \lambda} f(x)=\frac{1}{\Gamma(\tau)} \int_{x}^{+\infty} f(\xi)(\xi-x)^{\tau-1} e^{-\lambda(\xi-x)} d \xi .
$$


Definition 3 The positive and negative tempered equilibrium derivatives of order $0<\tau<$ 1 are defined as follows (see [27]):

$$
\begin{aligned}
& \mathbb{D}_{+}^{\tau, \lambda} f(x)=\lambda^{\tau} f(x)+\frac{\tau}{\Gamma(1-\tau)} \int_{-\infty}^{x} \frac{f(x)-f(\xi)}{(x-\xi)^{\tau+1}} e^{-\lambda(x-\xi)} d \xi, \\
& \mathbb{D}_{-}^{\tau, \lambda} f(x)=\lambda^{\tau} f(x)+\frac{\tau}{\Gamma(1-\tau)} \int_{x}^{+\infty} \frac{f(x)-f(\xi)}{(\xi-x)^{\tau+1}} e^{-\lambda(\xi-x)} d \xi,
\end{aligned}
$$

for any $\lambda>0$, respectively, where $f: \mathbb{R} \rightarrow \mathbb{R}$.

Define the Banach space

$$
W_{\lambda}^{\tau, 2}(\mathbb{R})=\left\{f \in L^{2}(\mathbb{R}): \int_{\mathbb{R}}\left(\lambda^{2}+\omega^{2}\right)^{\tau}|\widehat{f}(\omega)|^{2} d \omega<\infty\right\}
$$

with the norm

$$
\|f\|_{\tau, \lambda}=\left(\int_{\mathbb{R}}\left(\lambda^{2}+\omega^{2}\right)^{\tau}|\widehat{f}(\omega)|^{2} d \omega\right)^{1 / 2}
$$

For any $f \in W_{\lambda}^{\tau, 2}(\mathbb{R})$, let $\mathbb{D}_{ \pm}^{\tau, \lambda} f(x)$ denote the subfunctions associated with the universal equilibrium operator (1) with Fourier transform $(\lambda \pm i \omega)^{\tau} \widehat{f}(\omega)$ (see [23]), where the Fourier transform of $u(x)$ is defined as follows:

$$
\mathcal{F}(u)(\xi)=\int_{-\infty}^{\infty} e^{-i x \cdot \xi} u(x) d x .
$$

Now we state the following known results.

Lemma 1 (see [23])

(i)

$$
\mathbb{D}_{ \pm}^{\tau, \lambda} \mathbb{I}_{ \pm}^{\tau, \lambda} f(x)=f(x)
$$

for any $\tau, \lambda>0$ and $f \in L^{2}(\mathbb{R})$ and

$$
\mathbb{I}_{ \pm}^{\tau, \lambda} \mathbb{D}_{ \pm}^{\tau, \lambda} f(x)=f(x)
$$

for any $f \in W_{\lambda}^{\tau, 2}(\mathbb{R})$.

(ii)

$$
\left\langle f, \mathbb{D}_{+}^{\tau, \lambda} g\right\rangle_{L^{2}(\mathbb{R})}=\left\langle\mathbb{D}_{-}^{\tau, \lambda} f, g\right\rangle_{L^{2}(\mathbb{R})}
$$

for any $\tau, \lambda>0$ and $f, g \in W_{\lambda}^{\tau, 2}(\mathbb{R})$.

Lemma 2 (see [2])

(i) For any $\tau, \lambda>0$ and $p \geq 1, \mathbb{I}_{ \pm}^{\tau, \lambda}: L^{p}(\mathbb{R}) \rightarrow L^{p}(\mathbb{R})$ are bounded equilibrium operators with

$$
\left\|\mathbb{I}_{ \pm}^{\tau, \lambda} f\right\|_{L^{p}(\mathbb{R})} \leq \lambda^{-\tau}\|f\|_{L^{p}(\mathbb{R})}
$$


(ii)

$$
\begin{gathered}
\mathbb{I}_{ \pm}^{\tau, \lambda} \mathbb{I}_{ \pm}^{\beta, \lambda} f(x)=\mathbb{I}_{ \pm}^{\tau+\beta, \lambda} f(x) \\
\text { for any } \tau, \beta, \lambda>0 \text { and } f \in L^{p}(\mathbb{R}) .
\end{gathered}
$$

(iii)

$$
\begin{aligned}
& \qquad\left\langle f, \mathbb{I}_{+}^{\tau, \lambda} g\right\rangle_{L^{2}(\mathbb{R})}=\left\langle\mathbb{I}_{-}^{\tau, \lambda} f, g\right\rangle_{L^{2}(\mathbb{R})} \\
& \text { for any } \tau, \lambda>0 \text { and } f, g \in L^{2}(\mathbb{R}) \text {. }
\end{aligned}
$$

Next, for $0<\tau<1$, we define a fractional Sobolev space $H^{\tau}(\mathbb{R})$ as follows:

$$
H^{\tau}(\mathbb{R})={\overline{C_{0}^{\infty}(\mathbb{R})}}^{\|\cdot\|_{\tau}}
$$

endowed with

$$
\|u\|_{\tau}=\left(\int_{\mathbb{R}}|u(t)|^{2} d t+\int_{\mathbb{R}}|\omega|^{2 \tau}|\widehat{u}(\omega)|^{2} d \omega\right)^{1 / 2} .
$$

It follows that

$$
\begin{gathered}
2^{\frac{\tau-1}{2}}\|u\|_{\tau} \leq\|u\|_{\tau, 1} \leq\|u\|_{\tau} \\
\|u\|_{\tau, 1} \leq\|u\|_{\tau, \lambda} \leq \lambda^{\tau}\|u\|_{\tau, 1} \\
\|u\|_{\tau, \lambda}<\|u\|_{\tau, 1}<\lambda^{-\tau}\|u\|_{\tau, \lambda}
\end{gathered}
$$

for $0<\tau<1$, where $\|u\|_{\tau, 1}$ is the norm on $W_{1}^{\tau, 2}(\mathbb{R})$, and so $W_{1}^{\tau, 2}(\mathbb{R})=H^{\tau}(\mathbb{R})$ with equivalent norms.

Lemma 3 (see $[5,18]$ ) Let $\tau>1 / 2$. Then any $u \in W_{\lambda}^{\tau, 2}(\mathbb{R})$ is uniformly continuous, bounded and there exists a constant $C=C_{\tau}$ such that

$$
\sup _{t \in \mathbb{R}}|u(t)| \leq C\|u\|_{\tau, \lambda}
$$

Remark 2 From Lemma 3 and (5)-(7), we have the following implication: if $u \in W_{\lambda}^{\tau, 2}$ with $\frac{1}{2}<\tau<1$, then $u \in L^{q}(\mathbb{R})$ for all $q \in[2, \infty)$ as

$$
\int_{\mathbb{R}}|u(t)|^{q} d t \leq\|u\|_{\infty}^{q-2}\|u\|_{L^{2}(\mathbb{R})}^{2} \leq 2^{1-\tau} C^{q-2}\|u\|_{\tau, \lambda}^{q}
$$

Remark 3 The imbedding of $W_{\lambda}^{\tau, 2}$ in $L^{q}(-T, T)$ is compact for $q \in(2, \infty)$ and any $T>0$ (see [3]).

\section{Existence and convergence}

In this section, we prove the existence of solution of (3) and discuss the convergence of the sequence. 
Theorem 1 Assume that $u$ is a critical point of

$$
I(w):=\psi(w)-\frac{1}{p} \int_{B_{1}} a(|x|)|w|^{p} d x
$$

If there exists $v \in \operatorname{Dom}(\psi)$ satisfying the equilibrium equation

$$
\begin{aligned}
& -\Delta v+v=a(|x|)|u|^{p-2} u, \quad x \in B_{1} \\
& \frac{\partial v}{\partial v}=0, \quad x \in \partial B_{1},
\end{aligned}
$$

then $u$ is a meromorphic solution of (3).

Proof Assume that $\left\{\varrho_{n}\right\}_{n \in \mathbf{N}} \subset W_{\lambda}^{\tau, 2}(\mathbb{R})$ is a sequence such that $\left\{\Phi\left(\varrho_{n}\right)\right\}_{n \in \mathbb{N}}$ is bounded and $\Phi^{\prime}\left(\varrho_{n}\right) \rightarrow 0$ as $n \rightarrow \infty$. Then there exists a positive constant $D$ such that

$$
\left|\Phi\left(\varrho_{n}\right)\right| \leq D \quad \text { and } \quad\left\|\Phi^{\prime}\left(\varrho_{n}\right)\right\|_{\left(W_{\lambda}^{\tau, 2}(\mathbb{R})\right)^{*}} \leq D
$$

for any $n \in \mathbb{N}$, where $\left(W_{\lambda}^{\tau, 2}(\mathbb{R})\right)^{*}$ is the dual space of $W_{\lambda}^{\tau, 2}(\mathbb{R})$.

Firstly, we show that $\left\{\varrho_{n}\right\}_{n \in \mathbf{N}}$ is bounded. Without loss of generality, we assume that

$$
\inf _{n}\left\|\varrho_{n}\right\|_{\tau, \lambda}=\eta>0
$$

denote by $\varrho=\varrho(\eta)$ the number corresponding to $\delta=\eta^{2}$ in $(\mathrm{C} 1)$ such that

$$
M\left(\left\|\varrho_{n}\right\|_{\tau, \lambda}^{2}\right) \geq \varrho
$$

for all $n$.

In view of (C2) and (12), one gets

$$
\begin{aligned}
D+D\left\|\varrho_{n}\right\|_{\tau, \lambda} \geq & \Phi\left(\varrho_{n}\right)-\frac{1}{\mu} \Phi^{\prime}\left(\varrho_{n}\right) \varrho_{n} \\
= & \frac{1}{2} \widehat{M}\left(\left\|\varrho_{n}\right\|_{\tau, \lambda}^{2}\right)-\frac{1}{\mu} M\left(\left\|\varrho_{n}\right\|_{\tau, \lambda}^{2}\right)\left\|\varrho_{n}\right\|_{\tau, \lambda}^{2} \\
& -\frac{1}{\mu} \int_{\mathbb{R}}\left(\mu F\left(t, \varrho_{n}(t)\right)-f\left(t, \varrho_{n}(t)\right) \varrho_{n}(t)\right) d t \\
\geq & \left(\frac{1}{2 \Upsilon}-\frac{1}{\mu}\right) M\left(\left\|\varrho_{n}\right\|_{\tau, \lambda}^{2}\right)\left\|\varrho_{n}\right\|_{\tau, \lambda}^{2} \\
\geq & \varrho\left(\frac{1}{2 \Upsilon}-\frac{1}{\mu}\right)\left\|\varrho_{n}\right\|_{\tau, \lambda}^{2} .
\end{aligned}
$$

Since $\mu>2 \Upsilon$, the boundedness of $\left\{\varrho_{n}\right\}_{n \in \mathbf{N}}$ follows directly. So there exists a subsequence $\left\{\varrho_{n}\right\}_{n \in \mathbf{N}}$ and $u \in W_{\lambda}^{\tau, 2}$ such that

$$
\varrho_{n} \rightarrow u \quad \text { weakly in } W_{\lambda}^{\tau, 2}(\mathbb{R})
$$


which yields

$$
\begin{aligned}
\Phi^{\prime}\left(\varrho_{n}\right)\left(\varrho_{n}-\varrho\right)= & M\left(\left\|\varrho_{n}\right\|_{\tau, \lambda}^{2}\right) \int_{\mathbb{R}}\left(\mathbb{D}_{+}^{\tau, \lambda} \varrho_{n} \mathbb{D}_{+}^{\tau, \lambda}\left(\varrho_{n}-\varrho\right)\right) d t \\
& -\int_{\mathbb{R}} f\left(t, \varrho_{n}\right)\left(\varrho_{n}-\varrho\right) d t \rightarrow 0
\end{aligned}
$$

as $n \rightarrow \infty$

Now we show that

$$
\lim _{n \rightarrow \infty} \int_{\mathbb{R}} f\left(t, \varrho_{n}\right)\left(\varrho_{n}-\varrho\right) d t=0 .
$$

To this end, by (13), there exists some positive constant $d$ such that

$$
\begin{aligned}
& \left\|\varrho_{n}\right\|_{\tau, \lambda}<d \text { and }\|u\|_{\tau, \lambda}<d, \quad \text { for } n \in \mathbb{N}, \\
& \varrho_{n} \rightarrow u \text { strongly in } L^{q}(\mathbb{R}) \text { and a.e. in } \mathbb{R} .
\end{aligned}
$$

Moreover, (C4) implies that there exists a positive constant $T$ such that

$$
f\left(t, \varrho_{n}\right) \leq \varepsilon\left|\varrho_{n}\right|^{q-1}
$$

for any $\varepsilon>0$ and $|t|>T$.

Then, by using Remark 2 and Young's inequality, we obtain

$$
\begin{aligned}
& \left|\int_{\mathbb{R}} f\left(t, \varrho_{n}\right)\left(\varrho_{n}-\varrho\right) d t\right| \\
& \quad \leq \int_{\mathbb{R}}\left|f\left(t, \varrho_{n}\right)\right|\left|\varrho_{n}-u\right| d t \\
& \quad \leq \int_{-T}^{T}\left|f\left(t, \varrho_{n}\right)\right|\left|\varrho_{n}-u\right| d t+\int_{|t|>T}\left|f\left(t, \varrho_{n}\right)\right|\left|\varrho_{n}-u\right| d t \\
& \quad \leq \varepsilon\left\|\varrho_{n}\right\|_{\infty}+\varepsilon \int_{|t|>T}\left|\varrho_{n}\right|^{q-1}\left|\varrho_{n}-u\right| d t \\
& \quad \leq \varepsilon C\left\|\varrho_{n}\right\|_{\tau, \lambda}+\varepsilon \int_{|t|>T}\left(\frac{q-1}{q}\left|\varrho_{n}\right|^{q}+\frac{1}{\mu}\left|\varrho_{n}-u\right|^{q}\right) d t \\
& \quad \leq \varepsilon C\left\|\varrho_{n}\right\|_{\tau, \lambda}+\frac{q-1}{q} \varepsilon 2^{1-\tau} C^{q-2}\left\|\varrho_{n}\right\|_{\tau, \lambda}^{q}+\varepsilon \frac{1}{\mu} 2^{1-\tau} C^{q-2}\left\|\varrho_{n}-u\right\|_{\tau, \lambda}^{q-2} \\
& \quad \leq \varepsilon C d+\frac{q-1}{q} \varepsilon 2^{1-\tau} C^{q-2} d^{q}+\varepsilon \frac{1}{\mu} 2^{1-\tau} C^{q-2}\left\|\varrho_{n}-u\right\|_{\tau, \lambda}^{q-2}
\end{aligned}
$$

for large enough $n$ from (8).

Then

$$
\lim _{n \rightarrow \infty} \int_{\mathbb{R}} f\left(t, \varrho_{n}\right)\left(\varrho_{n}-\varrho\right) d t=0 .
$$


Therefore, we have

$$
M\left(\left\|\varrho_{n}\right\|_{\tau, \lambda}^{2}\right) \int_{\mathbb{R}}\left(\mathbb{D}_{+}^{\tau, \lambda} \varrho_{n} \mathbb{D}_{+}^{\tau, \lambda}\left(\varrho_{n}-\varrho\right)\right) d t \rightarrow 0
$$

from (14) as $n \rightarrow \infty$.

Thus, by the boundedness of $M\left(\left\|\varrho_{n}\right\|_{\tau, \lambda}^{2}\right)$, one can get

$$
\int_{\mathbb{R}}\left(\mathbb{D}_{+}^{\tau, \lambda} \varrho_{n} \mathbb{D}_{+}^{\tau, \lambda}\left(\varrho_{n}-\varrho\right)\right) d t \rightarrow 0
$$

from (12) as $n \rightarrow \infty$.

In a similar manner, we can get

$$
\int_{\mathbb{R}}\left(\mathbb{D}_{+}^{\tau, \lambda} u \mathbb{D}_{+}^{\tau, \lambda}\left(\varrho_{n}-\varrho\right)\right) d t \rightarrow 0
$$

as $n \rightarrow \infty$.

Combining (16) and (17), we obtain that

$$
\int_{\mathbb{R}}\left(\mathbb{D}_{+}^{\tau, \lambda}\left(\varrho_{n}-\varrho\right) \mathbb{D}_{+}^{\tau, \lambda}\left(\varrho_{n}-\varrho\right)\right) d t \rightarrow 0
$$

as $n \rightarrow \infty$.

Hence,

$$
\left\|\varrho_{n}-u\right\|_{\tau, \lambda} \rightarrow 0
$$

as $n \rightarrow \infty$, and then $\Phi$ satisfies the Palais-Smale condition.

Theorem 2 Let the following two conditions hold:

(1) $\forall \sigma_{i}, \varrho_{i} \in H_{i}, \eta_{i}\left(\sigma_{i}, \varrho_{i}\right)=-\eta_{i}\left(\varrho_{i}, \sigma_{i}\right)$;

(2) $a_{i}: H_{i} \times H_{i} \rightarrow \mathbb{R}$ satisfies (C1) and (C2), $b_{i}: H_{i} \times H_{i} \rightarrow \mathbb{R}$ with (C3)-(C6).

Moreover, we have the following conditions:

$$
\begin{aligned}
& 0<\frac{1}{\sigma_{1}+\rho_{1} c_{1}}\left[\delta \sqrt{1-2 \rho_{1} \tau_{1}+\rho_{1}^{2} \beta_{1}^{2}}+\rho_{1} \gamma_{1}\right]+\frac{\rho_{2} \delta_{2} \xi_{2}}{\sigma_{2}+\rho_{2} c_{2}}<1, \\
& 0<\frac{1}{\sigma_{2}+\rho_{2} c_{2}}\left[\delta_{2} \sqrt{1-2 \rho_{2} \tau_{2}+\rho_{2}^{2} \beta_{2}^{2}}+\rho_{2} \gamma_{2}\right]+\frac{\rho_{1} \delta_{1} \xi_{1}}{\sigma_{1}+\rho_{1} c_{1}}<1 .
\end{aligned}
$$

Then the sequence $\left\{\left(\varrho_{n}, \sigma_{n}\right)\right\}_{n \geq 0}$ converges to $\left(\varrho^{*}, \sigma^{*}\right)$, where $\left(\varrho^{*}, \sigma^{*}\right)$ is the meromorphic solution of (3).

Proof It follows from (18) and (19) that

$$
\begin{array}{r}
\left\langle\varrho_{n}-\varrho_{n-1}, \eta_{1}\left(\sigma_{1}, \varrho_{n}\right)\right\rangle_{1}+\rho_{1}\left\langle F_{1}\left(A \varrho_{n-1}, B \sigma_{n-1}\right)-f_{1}, \eta_{1}\left(\sigma_{1}, \varrho_{n}\right)\right\rangle_{1} \\
+\rho_{1}\left[a_{1}\left(\varrho_{n}, \sigma_{1}-\varrho_{n}\right)\right]+\rho_{1}\left[b_{1}\left(\varrho_{n-1}, \sigma_{1}\right)-b_{1}\left(\varrho_{n-1}, \varrho_{n}\right)\right] \geq 0, \\
\left\langle\sigma_{n}-\sigma_{n-1}, \eta_{2}\left(\sigma_{2}, \sigma_{n}\right)\right\rangle_{2}+\rho_{2}\left\langle F_{2}\left(A \varrho_{n-1}, B \sigma_{n-1}\right)-f_{2}, \eta_{2}\left(\sigma_{2}, \sigma_{n}\right)\right\rangle_{2} \\
+\rho_{2}\left[a_{2}\left(\sigma_{n}, \sigma_{2}-\sigma_{n}\right)\right]+\rho_{2}\left[b_{2}\left(\sigma_{n-1}, \sigma_{2}\right)-b_{1}\left(\sigma_{n-1}, \sigma_{n}\right)\right] \geq 0
\end{array}
$$

for any $\left(\sigma_{1}, \sigma_{2}\right) \in H_{1} \times H_{2}$. 
If we take $\sigma_{1}=\varrho_{n+1}$ in (20) and $\sigma_{1}=\varrho_{n}$ in (21), respectively, then

$$
\begin{aligned}
& \left\langle\varrho_{n}-\varrho_{n-1}, \eta_{1}\left(\varrho_{n+1}, \varrho_{n}\right)\right\rangle_{1}+\rho_{1}\left\langle F_{1}\left(A \varrho_{n-1}, B \sigma_{n-1}\right)-f_{1}, \eta_{1}\left(\varrho_{n+1}, \varrho_{n}\right)\right\rangle_{1} \\
& \quad+\rho_{1}\left[a_{1}\left(\varrho_{n}, \varrho_{n+1}-\varrho_{n}\right)\right]+\rho_{1}\left[b_{1}\left(\varrho_{n-1}, \varrho_{n+1}\right)-b_{1}\left(\varrho_{n-1}, \varrho_{n}\right)\right] \geq 0 \\
& \left\langle\varrho_{n+1}-\varrho_{n}, \eta_{1}\left(\varrho_{n}, \varrho_{n+1}\right)\right\rangle_{1}+\rho_{1}\left\langle F_{1}\left(A \varrho_{n}, B \sigma_{n}\right)-f_{1}, \eta_{1}\left(\varrho_{n}, \varrho_{n+1}\right)\right\rangle_{1} \\
& \quad+\rho_{1}\left[a_{1}\left(\varrho_{n+1}, \varrho_{n}-\varrho_{n+1}\right)\right]+\rho_{1}\left[b_{1}\left(\varrho_{n}, \varrho_{n}\right)-b_{1}\left(\varrho_{n}, \varrho_{n+1}\right)\right] \geq 0
\end{aligned}
$$

Adding (22) and (23), we obtain that

$$
\begin{aligned}
\left\langle\varrho_{n}-\right. & \left.\varrho_{n+1}, \eta_{1}\left(\varrho_{n}, \varrho_{n+1}\right)\right\rangle_{1} \\
\leq & \left\langle\varrho_{n-1}-\varrho_{n}, \eta_{1}\left(\varrho_{n}, \varrho_{n+1}\right)\right\rangle_{1}-\rho_{1}\left\langle F_{1}\left(A \varrho_{n-1}, B \sigma_{n-1}\right)-F_{1}\left(A \varrho_{n}, B \sigma_{n}\right), \eta_{1}\left(\varrho_{n}, \varrho_{n+1}\right)\right\rangle_{1} \\
& -\rho_{1}\left[a_{1}\left(\varrho_{n}-\varrho_{n+1}, \varrho_{n}-\varrho_{n+1}\right)\right]+\rho_{1}\left[b_{1}\left(\varrho_{n-1}-\varrho_{n}, \varrho_{n+1}\right)+b_{1}\left(\varrho_{n}-\varrho_{n-1}, \varrho_{n}\right)\right] \\
\leq & \left\langle\varrho_{n-1}-\varrho_{n}-\rho_{1}\left[F_{1}\left(A \varrho_{n-1}, B \sigma_{n-1}\right)-F_{1}\left(A \varrho_{n}, B \sigma_{n}\right)\right], \eta_{1}\left(\varrho_{n}, \varrho_{n+1}\right)\right\rangle_{1} \\
& -\rho_{1}\left[a_{1}\left(\varrho_{n}-\varrho_{n+1}, \varrho_{n}-\varrho_{n+1}\right)\right]+\rho_{1}\left[b_{1}\left(\varrho_{n}-\varrho_{n-1}, \varrho_{n}-\varrho_{n+1}\right)\right] .
\end{aligned}
$$

Since $\eta_{1}$ is $\sigma_{1}$-strongly monotone and $\delta_{1}$-Lipschitz continuous and $a_{1}$ satisfies $(\mathrm{C} 1)$, we have

$$
\begin{aligned}
& \sigma_{1}\left\|\varrho_{n}-\varrho_{n+1}\right\|_{1}^{2} \\
& \leq\left\|\varrho_{n-1}-\varrho_{n}-\rho_{1}\left[F_{1}\left(A \varrho_{n-1}, B \sigma_{n-1}\right)-F_{1}\left(A \varrho_{n}, B \sigma_{n}\right)\right]\right\|_{1}\left\|\eta_{1}\left(\varrho_{n}, \varrho_{n+1}\right)\right\|_{1} \\
&-\rho_{1} c_{1}\left\|\varrho_{n}-\varrho_{n+1}\right\|_{1}^{2}+\rho_{1} \gamma_{1}\left\|\varrho_{n}-\varrho_{n-1}\right\|_{1}\left\|\varrho_{n}-\varrho_{n+1}\right\|_{1} \\
& \leq \delta_{1}\left\|\varrho_{n-1}-\varrho_{n}-\rho_{1}\left[F_{1}\left(A \varrho_{n-1}, B \sigma_{n-1}\right)-F_{1}\left(A \varrho_{n}, B \sigma_{n}\right)\right]\right\|_{1}\left\|\varrho_{n}-\varrho_{n+1}\right\|_{1} \\
&-\rho_{1} c_{1}\left\|\varrho_{n}-\varrho_{n+1}\right\|_{1}^{2}+\rho_{1} \gamma_{1}\left\|\varrho_{n}-\varrho_{n-1}\right\|_{1}\left\|\varrho_{n}-\varrho_{n+1}\right\|_{1},
\end{aligned}
$$

which implies that

$$
\begin{aligned}
\left\|\varrho_{n}-\varrho_{n+1}\right\|_{1} & \\
\leq & \frac{1}{\sigma_{1}+\rho_{1} c_{1}}\left(\delta_{1}\left\|\varrho_{n-1}-\varrho_{n}-\rho_{1}\left[F_{1}\left(A \varrho_{n-1}, B \sigma_{n-1}\right)-F_{1}\left(A \varrho_{n}, B \sigma_{n-1}\right)\right]\right\|_{1}\right. \\
& \left.+\rho_{1} \delta_{1}\left\|F_{1}\left(A \varrho_{n}, B \sigma_{n-1}\right)-F_{1}\left(A \varrho_{n}, B \sigma_{n}\right)\right\|_{1}+\rho_{1} \gamma_{1}\left\|\varrho_{n}-\varrho_{n-1}\right\|_{1}\right) .
\end{aligned}
$$

So

$$
\begin{aligned}
&\left\|\varrho_{n-1}-\varrho_{n}-\rho_{1}\left[F_{1}\left(A \varrho_{n-1}, B \sigma_{n-1}\right)-F_{1}\left(A \varrho_{n}, B \sigma_{n-1}\right)\right]\right\|_{1}^{2} \\
&=\left\|\varrho_{n-1}-\varrho_{n}\right\|_{1}^{2}-2 \rho_{1}\left\langle F_{1}\left(A \varrho_{n-1}, B \sigma_{n-1}\right)-F_{1}\left(A \varrho_{n}, B \sigma_{n-1}\right), \varrho_{n-1}-\varrho_{n}\right\rangle_{1} \\
& \quad+\rho_{1}^{2}\left\|F_{1}\left(A \varrho_{n-1}, B \sigma_{n-1}\right)-F_{1}\left(A \varrho_{n}, B \sigma_{n-1}\right)\right\|_{1}^{2} \\
& \leq\left(1-2 \rho_{1} \tau_{1}+\rho_{1}^{2} \beta_{1}^{2}\right)\left\|\varrho_{n-1}-\varrho_{n}\right\|_{1}^{2}
\end{aligned}
$$

and

$$
\left\|F_{1}\left(A \varrho_{n}, B \sigma_{n-1}\right)-F_{1}\left(A \varrho_{n}, B \sigma_{n}\right)\right\|_{1} \leq \xi_{1}\left\|\sigma_{n-1}-\sigma_{n}\right\|_{2} .
$$


It follows from (24), (25), and (26) that

$$
\begin{aligned}
\left\|\varrho_{n}-\varrho_{n+1}\right\|_{1} \leq & \frac{1}{\sigma_{1}+\rho_{1} c_{1}}\left[\delta \sqrt{1-2 \rho_{1} \tau_{1}+\rho_{1}^{2} \beta_{1}^{2}}+\rho_{1} \gamma_{1}\right]\left\|\varrho_{n-1}-\varrho_{n}\right\|_{1} \\
& +\frac{\rho_{1} \delta_{1} \xi_{1}}{\sigma_{1}+\rho_{1} c_{1}}\left\|\sigma_{n-1}-\sigma_{n}\right\|_{2}
\end{aligned}
$$

taking $\sigma_{2}=\sigma_{n+1}$ in (25) and $\sigma_{2}=\sigma_{n}$ in (26), respectively.

Similarly, we have

$$
\begin{aligned}
\left\|\sigma_{n}-\sigma_{n+1}\right\|_{2} \leq & \frac{1}{\sigma_{2}+\rho_{2} c_{2}}\left[\delta_{2} \sqrt{1-2 \rho_{2} \tau_{2}+\rho_{2}^{2} \beta_{2}^{2}}+\rho_{2} \gamma_{2}\right]\left\|\sigma_{n-1}-\sigma_{n}\right\|_{2} \\
& +\frac{\rho_{2} \delta_{2} \xi_{2}}{\sigma_{2}+\rho_{2} c_{2}}\left\|\varrho_{n-1}-\varrho_{n}\right\|_{1} .
\end{aligned}
$$

From (27) and (28), we obtain that

$$
\begin{aligned}
&\left\|\varrho_{n}-\varrho_{n+1}\right\|_{1}+\left\|\sigma_{n}-\sigma_{n+1}\right\|_{2} \\
& \leq\left(\frac{1}{\sigma_{1}+\rho_{1} c_{1}}\left[\delta_{1} \sqrt{1-2 \rho_{1} \tau_{1}+\rho_{1}^{2} \beta_{1}^{2}}+\rho_{1} \gamma_{1}\right]+\frac{\rho_{2} \delta_{2} \xi_{2}}{\sigma_{2}+\rho_{2} c_{2}}\right)\left\|\varrho_{n-1}-\varrho_{n}\right\|_{1} \\
&+\left(\frac{1}{\sigma_{2}+\rho_{2} c_{2}}\left[\delta_{2} \sqrt{1-2 \rho_{2} \tau_{2}+\rho_{2}^{2} \beta_{2}^{2}}+\rho_{2} \gamma_{2}\right]+\frac{\rho_{1} \delta_{1} \xi_{1}}{\sigma_{1}+\rho_{1} c_{1}}\right)\left\|\sigma_{n-1}-\sigma_{n}\right\|_{2} \\
& \leq \max \left\{\theta_{1}, \theta_{2}\right\}\left(\left\|\varrho_{n-1}-\varrho_{n}\right\|_{1}+\left\|\sigma_{n-1}-\sigma_{n}\right\|_{2}\right),
\end{aligned}
$$

where

$$
\begin{aligned}
& \theta_{1}:=\frac{1}{\sigma_{1}+\rho_{1} c_{1}}\left[\delta_{1} \sqrt{1-2 \rho_{1} \tau_{1}+\rho_{1}^{2} \beta_{1}^{2}}+\rho_{1} \gamma_{1}\right]+\frac{\rho_{2} \delta_{2} \xi_{2}}{\sigma_{2}+\rho_{2} c_{2}}, \\
& \theta_{2}:=\frac{1}{\sigma_{2}+\rho_{2} c_{2}}\left[\delta_{2} \sqrt{1-2 \rho_{2} \tau_{2}+\rho_{2}^{2} \beta_{2}^{2}}+\rho_{2} \gamma_{2}\right]+\frac{\rho_{1} \delta_{1} \xi_{1}}{\sigma_{1}+\rho_{1} c_{1}} .
\end{aligned}
$$

Now, if we define the norm $\|\cdot\|_{*}$ on $H_{1} \times H_{2}$ by

$$
\|(u, v)\|_{*}=\|u\|_{1}+\|v\|_{2}
$$

for any $(u, v) \in H_{1} \times H_{2}$, then we have

$$
\left\|\left(\varrho_{n}, \sigma_{n}\right)-\left(\varrho_{n+1}, \sigma_{n+1}\right)\right\|_{*} \leq \max \left\{\theta_{1}, \theta_{2}\right\}\left\|\left(\varrho_{n-1}, \sigma_{n-1}\right)-\left(\varrho_{n}, \sigma_{n}\right)\right\|_{*} .
$$

By using (18) and (19), it follows that $\theta_{1}, \theta_{2} \in(0,1)$. Hence, (30) implies that $\left\{\left(\varrho_{n}, \sigma_{n}\right)\right\}$ is a Halton sequence in $H_{1} \times H_{2}$.

Let $\left(\varrho_{n}, \sigma_{n}\right) \rightarrow\left(\varrho^{*}, \sigma^{*}\right)$ in $H_{1} \times H_{2}$ as $n \rightarrow \infty$. Therefore,

$$
\begin{aligned}
& \left\langle F_{1}\left(A \varrho^{*}, B \sigma^{*}\right)-f_{1}, \eta_{1}\left(\sigma_{1}, \varrho^{*}\right)\right\rangle_{1}+a_{1}\left(\varrho^{*}, \sigma_{1}-\varrho^{*}\right)+b_{1}\left(\varrho^{*}, \sigma_{1}\right)-b_{1}\left(\varrho^{*}, \varrho^{*}\right) \geq 0 \\
& \quad \forall \sigma_{1} \in H_{1}, \\
& \left\langle F_{2}\left(A \varrho^{*}, B \sigma^{*}\right)-f_{2}, \eta_{2}\left(\sigma_{2}, \sigma^{*}\right)\right\rangle_{2}+a_{2}\left(\sigma^{*}, \sigma_{2}-\sigma^{*}\right)+b_{2}\left(\sigma^{*}, \sigma_{2}\right)-b_{2}\left(\sigma^{*}, \sigma^{*}\right) \geq 0 \\
& \quad \forall \sigma_{2} \in H_{2} .
\end{aligned}
$$


Thus, $\left(\varrho^{*}, \sigma^{*}\right)$ is a meromorphic solution of the model problem (3), which implies the required conclusion.

\section{Conclusions}

In this paper, we studied the equilibrium system with angular velocity for the prey. This system was a generalization of the two-species equilibrium model with Neumann type boundary condition. Firstly, we considered the asymptotical stability of equilibrium points to the system of ordinary differential equations type. Then, the existence of meromorphic solutions and the stability of equilibrium points to the system of weakly coupled meromorphic type were discussed. Finally, the existence of nonnegative meromorphic solutions to the system of strongly coupled meromorphic type was investigated, and the asymptotic stability of unique positive equilibrium point of the system was proved by constructing meromorphic functions.

\section{Acknowledgements}

Not applicable.

Funding

This work was supported by the Post-Doctoral Applied Research Projects of Qingdao (no. 2015122) and the Scientific Research Foundation of Shandong University of Science and Technology for Recruited Talents (no. 2014RCJJ032).

\section{Abbreviations}

Not applicable.

Availability of data and materials

Not applicable.

Competing interests

The author declares that he has no competing interests.

Authors' contributions

The author read and approved the final manuscript.

\section{Publisher's Note}

Springer Nature remains neutral with regard to jurisdictional claims in published maps and institutional affiliations.

Received: 5 February 2019 Accepted: 16 April 2019 Published online: 07 May 2019

\section{References}

1. Cui, Y.: Uniqueness of solution for boundary value problems for fractional differential equations. Appl. Math. Lett. 51, 48-54 (2016)

2. Cui, Y., Zou, Y.: Existence of solutions for second-order integral boundary value problems. Nonlinear Anal., Model. Control 21(6), 828-838 (2016)

3. Glowinski, R., Lions, J., Trémolières, R.: Numerical Analysis of Variational Inequalities. North-Holland, Amsterdam (1981)

4. Hartman, P., Stampacchia, G.: On some nonlinear elliptic differential functional equations. Acta Math. 115, 153-188 (1966)

5. Barta, T:: Convergence to equilibrium of relatively compact solutions to equilibrium equations. Asymptot. Anal. 4(81), 1-9 (2011)

6. Noor, M.: Mixed variational-like inequalities. Commun. Appl. Nonlinear Anal. 1, 63-75 (1994)

7. Panagiotopoulos, P.: Inequality Problems in Mechanics and Applications. Birkhäuser, Boston (1985)

8. Lions, J., Stampaccia, G.: Variational inequalities. Commun. Pure Appl. Math. 20, 493-512 (1967)

9. Kassay, G., Radulescu, V.D.: Equilibrium Problems and Applications. Mathematics in Science and Engineering. Elsevier/Academic Press, London (2018)

10. Chergui, L.: An existence and uniqueness theorem for a second order nonlinear system with coupled integral boundary value conditions. Appl. Math. Comput. 256, 438-444 (2015)

11. Cui, Y., Zou, Y.: Monotone iterative method for differential systems with coupled integral boundary value problems. Bound. Value Probl. 2013, 245 (2013)

12. Sheng, K., Zhang, W., Bai, Z.: Positive solutions to fractional boundary-value problems with p-Laplacian on time scales. Bound. Value Probl. 2018, Article ID 70 (2018)

13. Zhai, C., Wang, W., Li, H.: A uniqueness method to a new Hadamard fractional differential system with four-point boundary conditions. J. Inequal. Appl. 2018, Article ID 207 (2018)

14. Zhang, Y.: Existence results for a coupled system of nonlinear fractional multi-point boundary value problems at resonance. J. Inequal. Appl. 2018, Article ID 198 (2018) 
15. Wu, J., Zhang, X., Liu, L., Wu, Y., Cui, Y.: The convergence analysis and error estimation for unique solution of a p-Laplacian fractional differential equation with singular decreasing nonlinearity. Bound. Value Probl. 2018, Article ID $82(2018)$

16. Sun, Q., Ji, H., Cui, Y.: Positive solutions for boundary value problems of fractional differential equation with integral boundary conditions. J. Funct. Spaces 2018, Article ID 6461930 (2018)

17. Li, K., Li, J., Wang, W.: Epidemic reaction-diffusion systems with two types of boundary conditions. Electron. J. Differ. Equ. 2018(170), 1 (2018)

18. Bai, Z., Sun, W.: Existence and multiplicity of positive solutions for singular fractional boundary value problems. Comput. Math. Appl. 63(9), 1369-1381 (2012)

19. Wang, Y., Liu, Y., Cui, Y: Multiple sign-changing solutions for nonlinear fractional Kirchhoff equations. Bound. Value Probl. 2018, Article ID 193 (2018)

20. Ardila, A.: Orbital stability of standing waves for a system of nonlinear Schrödinger equations with three wave interaction. Nonlinear Anal. 167, 1-20 (2018)

21. Hu, J., Yin, H.: Nonlinear stability of rarefaction waves for the compressible Navier-Stokes equations with zero heat conductivity. Nonlinear Anal. 174, 242-277 (2018)

22. Alleche, B., Radulescu, V.D.: Further on set-valued equilibrium problems in the pseudo-monotone case and applications to Browder variational inclusions. Optim. Lett. 12(8), 1789-1810 (2018)

23. Bai, Z.: Eigenvalue intervals for a class of fractional boundary value problem. Comput. Math. Appl. 64(10), 3253-3257 (2012)

24. Cui, Y., Sun, J.: On existence of positive solutions of coupled integral boundary value problems for a nonlinear singular superlinear differential system. Electron. J. Qual. Theory Differ. Equ. 2012, Article ID 41 (2012)

25. Cui, Y.: Existence of solutions for coupled integral boundary value problem at resonance. Publ. Math. (Debr.) 89(1-2), 73-88 (2016)

26. Song, Q., Bai, Z:: Positive solutions of fractional differential equations involving the Riemann-Stieltjes integral boundary condition. Adv. Differ. Equ. 2018, Article ID 183 (2018)

27. Djafari, B., Khatibzadeh, H.: A strong convergence theorem for solutions to a nonhomogeneous second order equilibrium equation. J. Math. Anal. Appl. 363(2), 648-654 (2008)

\section{Submit your manuscript to a SpringerOpen ${ }^{\circ}$ journal and benefit from:}

- Convenient online submission

- Rigorous peer review

- Open access: articles freely available online

- High visibility within the field

- Retaining the copyright to your article

Submit your next manuscript at $\gg$ springeropen.com 